07,01

\title{
Анализ изменения диффузионных свойств неравновесных границ зерен при рекристаллизации и сверхпластической деформации субмикрокристаллических металлов и сплавов
}

\author{
(С) В.Н. Чувильдеев ${ }^{1}$, А.В. Нохрин ${ }^{1, \uparrow}$, О.Э. Пирожникова ${ }^{1}$, М.Ю. Грязнов $^{1}$, \\ Ю.Г. Лопатин ${ }^{1}$, М.М. Мышляев ${ }^{2,3}$, В.И. Копылов ${ }^{1,4}$ \\ ${ }^{1}$ Научно-исследовательский физико-технический институт \\ Национального исследовательского Нижегородского государственного университета им. Н.И. Лобачевского, \\ Нижний Новгород, Россия \\ ${ }^{2}$ Институт фоизики твердого тела РАН, \\ Черноголовка, Россия \\ ${ }^{3}$ Институт металлургии и материаловедения им. А.А. Байкова РАН, \\ Москва, Россия \\ ${ }^{4}$ Физико-технический институт НАН Беларуси, \\ Минск, Беларусь \\ ฯ E-mail: nokhrin@nifti.unn.ru
}

(Поступила в Редакцию 21 ноября 2016 г.

В окончательной редакции 14 фревраля 2017 г.)

\begin{abstract}
Описаны результаты исследований эффекта повышения коэффициента зернограничной диффузии при рекристаллизации и сверхпластической деформации субмикрокристаллических (СМК) материалов, полученных методами интенсивного пластического деформирования. Показано, что коэффициент зернограничной диффузии СМК-материалов зависит от интенсивности потока решеточных дислокаций, величина которого пропорциональна скорости миграции границ зерен при отжиге СМК-металлов или скорости внутризеренной деформации в условиях сверхпластической деформации СМК-сплавов. Установлено, что при высокой скорости миграции границ зерен или повышенных скоростей сверхпластической деформации интенсивность потока решеточных дислокаций, бомбардирующих границы зерен СМК-материала, превосходит интенсивность их диффузионной аккомодации, что приводит к увеличению коэффициента зернограничной диффузии и снижению энергии активации. Результаты численных расчетов сопоставлены с экспериментальными данными. Показано их хорошее согласие.
\end{abstract}

Работа выполнена при поддержке РФФИ (гранты № 15-03-08969, 15-08-09298) и частичной поддержке Министерства образования и науки РФ (проект № 2014/134 в рамках государственного задания вузам).

DOI: $10.21883 /$ FTT.2017.08.44759.420

\section{1. Введение}

В работах [1-3] было показано, что в процессе дорекристаллизационного отжига субмикрокристаллических (СМК) металлов, полученных методами интенсивного пластического деформирования, наблюдается возврат диффузионных свойств неравновесных границ зерен (НГЗ): увеличение энергии активации $Q_{b}^{*}$ и уменьшение коэффициента зернограничной диффузии $D_{b}^{*}$ от значений, характерных для неравновесных границ зерен и в некоторых случаях близких к параметрам расплава $\left(D_{b}^{*} \approx D_{L}, Q_{b}^{*} \approx Q_{L}\right)$, до равновесных значений $\left(D_{b}^{*} \approx D_{b}, Q_{b}^{*} \approx Q_{b}\right)$. Скорость процесса возврата диффузионных свойств неравновесных границ зерен СМК-металлов контролируется кинетикой процесса делокализации дислокаций ориентационного несоответствия (ДОН) в границах зерен, а в более крупнозернистых материалах - кинетикой процесса диффузионного „ухода“ скользящих компонент делокализованных дислокаций [1-3].

В соответствии с этим следовало бы ожидать, что диффузионные свойства границ зерен СМК-металлов при температурах отжига выше температуры начала рекристаллизации будут соответствовать диффузионным свойствам равновесных границ зерен. Тем не менее анализ экспериментальных данных по исследованию процессов миграции границ зерен при отжиге СМК-сплавов [4-9], a также процессов сверхпластической деформации СМК-металлов при повышенных температурах $[10,11]$ показывает, что диффузионные свойства границ зерен в некоторых случаях весьма значительно отличаются от равновесных. Следует также отметить ряд работ, в которых наблюдалась немонотонная зависимость коэффициента зернограничной диффузии $D_{b}$ и энергии активации $Q_{b}$ от температуры нагрева [4,6,8,12-14].

Непостоянство значений энергии активации $Q_{b}$ также было обнаружено при анализе экспериментальных результатов исследования процессов ползучести [15], сверхпластичности $[16,17]$ и внутреннего трения $[18,19]$.

Причины такого поведения остаются неясными и, по мнению некоторых авторов, связаны с влиянием процесса миграции границ зерен на их диффузионные свойства при отжиге СМК-материалов [20]. 
Таблица 1. Значения параметров, используемых для расчетов $[3,22,23]$

\begin{tabular}{|c|c|c|c|c|}
\hline \multirow{2}{*}{ Параметр } & \multirow{2}{*}{ Обозначение } & \multirow{2}{*}{$\begin{array}{c}\text { Единицы } \\
\text { измерения }\end{array}$} & \multicolumn{2}{|c|}{ Значение } \\
\hline & & & $\mathrm{Cu}$ & $\mathrm{Al}$ \\
\hline Вектор Бюргерса & $b$ & $\mathrm{~m}$ & $2.56 \cdot 10^{-10}$ & $2.86 \cdot 10^{-10}$ \\
\hline Атомный объем & $\Omega$ & $\mathrm{m}^{3}$ & $1.18 \cdot 10^{-29}$ & $1.66 \cdot 10^{-29}$ \\
\hline Характерный размер зерна & $d$ & $b$ & $2 \cdot 10^{3}$ & $4 \cdot 10^{3}$ \\
\hline Ширина границы зерна & $\delta$ & $b$ & & \\
\hline Модуль сдвига & $G$ & $\mathrm{GPa}$ & 43.6 & 25 \\
\hline Температура плавления & $T_{m}$ & $\mathrm{~K}$ & 1356 & 933 \\
\hline Отношение $\lambda \rho b^{3} / k T_{m}$ & $\lambda \rho b^{3} / k T_{m}$ & - & 1.15 & 1.96 \\
\hline Скачок объема при плавлении & $\Delta v_{m}$ & - & \multicolumn{2}{|c|}{$5 \cdot 10^{-2}$} \\
\hline Энтальпия поверхности жидкость-кристалл & $\gamma_{S / L}^{0} b^{2} / k T_{m}$ & & 0.900 & 1.160 \\
\hline Энтропия поверхности жидкость-кристалл & $S_{S / L} b^{2} / k$ & - & 0.767 & 0.920 \\
\hline Относительный свободный объем границ зерен & $\alpha$ & - & 0.36 & 0.38 \\
\hline Критический свободный объем границ зерен & $\alpha^{*}$ & - & \multicolumn{2}{|c|}{0.50} \\
\hline Избыточный свободный объем границ зерен & $\Delta \alpha$ & - & \multicolumn{2}{|c|}{$0.001,01$} \\
\hline Свободная энергия границы зерна & $\gamma_{b} b^{2} / k T_{m}$ & - & 1.93 & 2.125 \\
\hline Свободная (поверхностная) энергия „сухих“ участков границы & $\gamma_{0} b^{2} / k T_{m}$ & - & 1.95 & 1.50 \\
\hline Энтальпия границы зерна & $\gamma_{b}^{0} b^{2} / k T_{m}$ & - & 4.32 & 4.98 \\
\hline Энтропия границы зерна & $S_{b} b^{2} / k$ & - & 2.30 & 2.76 \\
\hline Предэкспоненциальный множитель коэффициента зернограничной диффузии & $\delta D_{b 0}$ & $\mathrm{~m}^{3} / \mathrm{s}$ & $5.0 \cdot 10^{-15}$ & $5.0 \cdot 10^{-14}$ \\
\hline Энергия активации зернограничной самодиффузии & $Q_{b}$ & $k T_{m}$ & 9.2 & 10.8 \\
\hline Предэкспоненциальный множитель коэффициента диффузии в расплаве & $D_{L 0}$ & $\mathrm{~m}^{2} / \mathrm{s}$ & $14.6 \cdot 10^{-8}$ & $14.9 \cdot 10^{-8}$ \\
\hline Энергия активации диффузии в расплаве & $Q_{L}$ & $k T_{m}$ & 3.6 & 3.8 \\
\hline
\end{tabular}

Целью настоящей работы является построение модели, описывающей зависимость параметров зернограничной диффузии от температурно-временнб́х условий рекристаллизационного отжига и температурно-скоростных условий сверхпластической деформации СМК-материалов.

\section{2. Эффект повышения коэффиициента зернограничной диффузии при миграции границ}

2.1. Описание модели. В соответствии с представлениями $[3,21]$ энергия активации $Q_{b}^{*}$ и коэффициент зернограничной диффузии $D_{b}^{*}$ зависят от степени неравновесности границ зерен, которая определяется плотностью внесенных в границы дефектов: дислокаций ориентационного несоответствия с вектором Бюргерса $\Delta b$ и плотностью $\rho_{b}$ и скользящих компонент делокализованных дислокаций с плотностью вектора Бюргерса $w_{t}$.

Особенно сложным представляется решение задачи о поведении зависимости $Q_{b}^{*}(T)$ и $D_{b}^{*}(T)$ в случае одновременного протекания процессов возврата и рекристаллизации. Как известно, мигрирующие границы зерен „заметают“ распределенные в зернах дислокации и обеспечивают тем самым поток дефектов, активно „бомбардирующих“ границы зерен и изменяющих уровень их неравновесности [3,21].

При рекристаллизации (росте зерен) поток дислокаций на границу зерна $I$ пропорционален скорости мигра- ции границ зерен $V_{m}$ и плотности решеточных дислокаций $\rho_{v}$, заметаемых движущейся границей: $I=\xi_{m} V_{m} b \rho_{v}$, где $\xi_{m}-$ доля дислокаций одного знака, попадающих в мигрирующую границу зерна $\left(\xi_{m}=10^{-4}-10^{-2}\right)$, $b$ - вектор Бюргерса решеточной дислокации $[1,3]$.

В этом случае кинетические уравнения, описывающие скорость накопления дефектов на границах зерен СМК-материалов, могут быть представлены в виде

$$
\begin{gathered}
\Delta b \dot{\rho}_{b}=\xi_{m} V_{m} b \rho_{v}-\frac{\Delta b \rho_{b}}{t_{1}}, \\
\dot{w}_{t}=\xi_{m} V_{m} b \rho_{v}-\frac{w_{t}}{t_{2}},
\end{gathered}
$$

где $t_{1}$ - характерное время процесса делокализации ДОН, $t_{2}$ - характерное время диффузионного „ухода“ скользящих компонент делокализованных дислокаций. Величины $t_{1}$ и $t_{2}$ в соответствии с теорией НГЗ могут быть рассчитаны с помощью следующих уравнений [3]:

$$
\begin{aligned}
& t_{1}=\frac{A_{1}}{\left(\rho_{b} \Delta b\right)^{3}} \frac{k T}{G \Omega} \frac{b^{3}}{\delta D_{b}}, \\
& t_{2}=\frac{(d / b)^{2}}{C_{1} w_{t}} \frac{k T}{G \Omega} \frac{b^{3}}{\delta D_{b}},
\end{aligned}
$$

где $A_{1}=10$ и $C_{1}=50$ - численные константы, $d-$ размер зерна, $\delta=2 b$ - ширина границы зерна, $G-$ модуль сдвига, $D_{b}$ - коэффициент диффузии по равновесным границам зерен, $k$ - постоянная Больцмана (табл. 1). 
В том случае, когда скорость миграции границ зерен $V_{m}$ при отжиге СМК-материала превышает величину $V_{m}^{*}$, следует ожидать повышения коэффициента зернограничной диффузии. В случае, если $V_{m}<V_{m}^{*}$, коэффициент зернограничной диффузии $D_{b}^{*}$ останется близким к его равновесному значению $D_{b}$.

В условиях быстрой миграции границ зерен $\left(V_{m} \geq V_{m}^{*}\right)$ уравнения для стационарной плотности ДОН и скользящих компонент делокализованных дислокаций удобно представить в виде [1]

$$
\begin{gathered}
\rho_{b}^{\mathrm{st}} \Delta b=\left(g_{1} \rho_{v} V_{m}\right)^{1 / 4}, \\
w_{t}^{\mathrm{st}}=d\left(g_{2} \rho_{v} V_{m}\right)^{1 / 2},
\end{gathered}
$$

где $g_{1}, g_{2}$ - параметры материала.

Как следует из анализа формул (5), (6), в силу зависимости $w_{t}$ от $d$, если размеры зерен малы $\left(d<d_{1}\right)$ или низка температура отжига $\left(T<T^{*}\right)$, в границах зерен преимущественно будут накапливаться ДОН $\left(\rho_{b} \Delta b \gg w_{t}\right)$. При высокой температуре $\left(T>T^{*}\right)$ или больших размерах зерен $\left(d>d_{1}\right)$ в границах будут преобладать скользящие компоненты делокализованных дислокаций $\left(w_{t} \gg \rho_{b} \Delta b\right)$.

Величина критического размера зерна $d_{1}$ в условиях миграции границ зерен может быть оценена исходя из соотношения $\rho_{b}^{\text {st }} \Delta b=w_{t}^{\text {st. }}$.

$$
d_{1}=\left[\left(\frac{A_{1} C_{1}^{2}}{\xi_{m}}\right)\left(\frac{\delta D_{b}}{\rho_{v} V_{m}}\right)\left(\frac{G \Omega}{k T}\right)\right]^{1 / 4} .
$$

Оценим характерные плотности дефектов в границах зерен и скорости миграции, при которых возможно повышение коэффициента зернограничной диффузии. Для этого необходимо, чтобы интенсивность потока „падающих“ в границу зерна дислокаций $\left(I^{+}=\xi_{m} \rho_{v} V_{m}\right)$ была больше интенсивности „ухода“ дефектов из границ вследствие развития процессов возврата $\left(I^{-}=\rho_{b} / t_{1}\right.$, $\left.I^{-}=w_{t} / t_{2} b\right)$. Приравняв эти интенсивности, можно определить значение $V_{m}^{*}$, при котором возможно повышение коэффициента зернограничной диффузии $D_{b}^{*}$ :

$$
\begin{aligned}
V_{m 1}^{*} & =\frac{\rho_{b}^{\mathrm{st}}}{\rho_{v} t_{1} \xi_{m}}, \\
V_{m 2}^{*} & =\frac{w_{t}^{\mathrm{st}}}{\rho_{v} t_{2} b \xi_{m}} .
\end{aligned}
$$

При начальной плотности дефектов $\rho_{b} \Delta b=10^{-3}$ и $w_{t}=10^{-3}$ [1] характерное время $t_{1}$, вычисленное по формуле (3), для СМК-меди при $T \sim 0.385 T_{m}(523 \mathrm{~K})$, где $T_{m}=1356 \mathrm{~K}$ - температура плавления, составляет $t_{1} \sim 5.9 \cdot 10^{3} \mathrm{~s}$. Величина критической скорости миграции границ зерен СМК-меди $V_{m 1}^{*}$, полученная по формуле (8) при $\rho_{v}=10^{14} \mathrm{~m}^{-2}$ и $T=523 \mathrm{~K}$, составляет $\sim 1.3 \cdot 10^{-9} \mathrm{~m} / \mathrm{s}$.

Величина $t_{2}$ для СМК-меди, вычисленная по формуле (4) при той же температуре и $d=0.2 \mu \mathrm{m}$, рав- на $t_{2}=89 \mathrm{~s}$, а величина критической скорости миграции $V_{m 2}^{*}$, найденная по формуле (9), составляет $\sim 1.4 \cdot 10^{-16} \mathrm{~m} / \mathrm{s}$.

Аналогичные результаты могут быть получены и для СМК-алюминия: при $T=373 \mathrm{~K} \sim 0.4 T_{m}\left(\rho_{b} \Delta b=10^{-3}\right.$, $\left.w_{t}=10^{-3}, \rho_{v}=10^{14} \mathrm{~m}^{-2}, d=1 \mu \mathrm{m}\right)$ характерные значения $t_{1}$ и $V_{m 1}^{*}$, вычисленные по формулам (3) и (8), будут составлять $3.2 \cdot 10^{4} \mathrm{~s}$ и $2.2 \cdot 10^{-10} \mathrm{~m} / \mathrm{s}$ соответственно, а значения $t_{2}$ и $V_{m 2}^{*}$, вычисленные по формулам (4) и (9), будут равны $0.47 \mathrm{~s}$ и $2.1 \cdot 10^{-15} \mathrm{~m} / \mathrm{s}$ соответственно.

Полученные оценки $\left(t_{1} \gg t_{2}\right)$ подтверждают ранее сделанное предположение о том, что в границах зерен СМК-материалов (при малом размере зерна и/или низких температурах отжига) доминируют ДОН $\left(\rho_{b}^{\mathrm{st}} \Delta b \gg w_{t}^{\mathrm{st}}\right)$.

Зависимости критической скорости миграции границ зерен $V_{m 1}^{*}$ от температуры отжига для СМК-меди и СМК-алюминия при различных начальных плотностях решеточных дислокаций представлены на рис. 1.
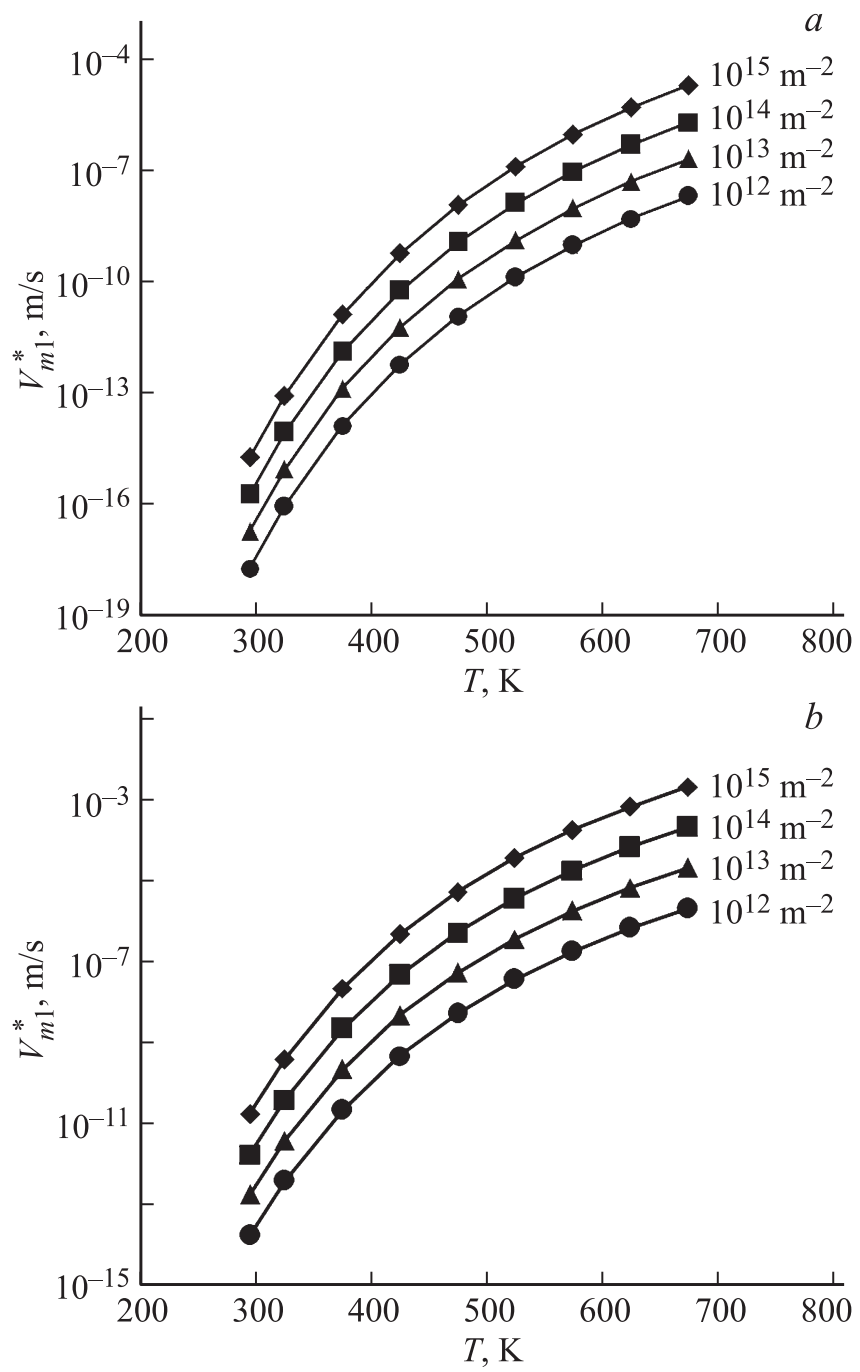

Рис. 1. Зависимости критической скорости миграции границ зерен $V_{m 1}^{*}$ от температуры отжига для СМК-меди $(a)$ и СМК-алюминия $(b)$. 

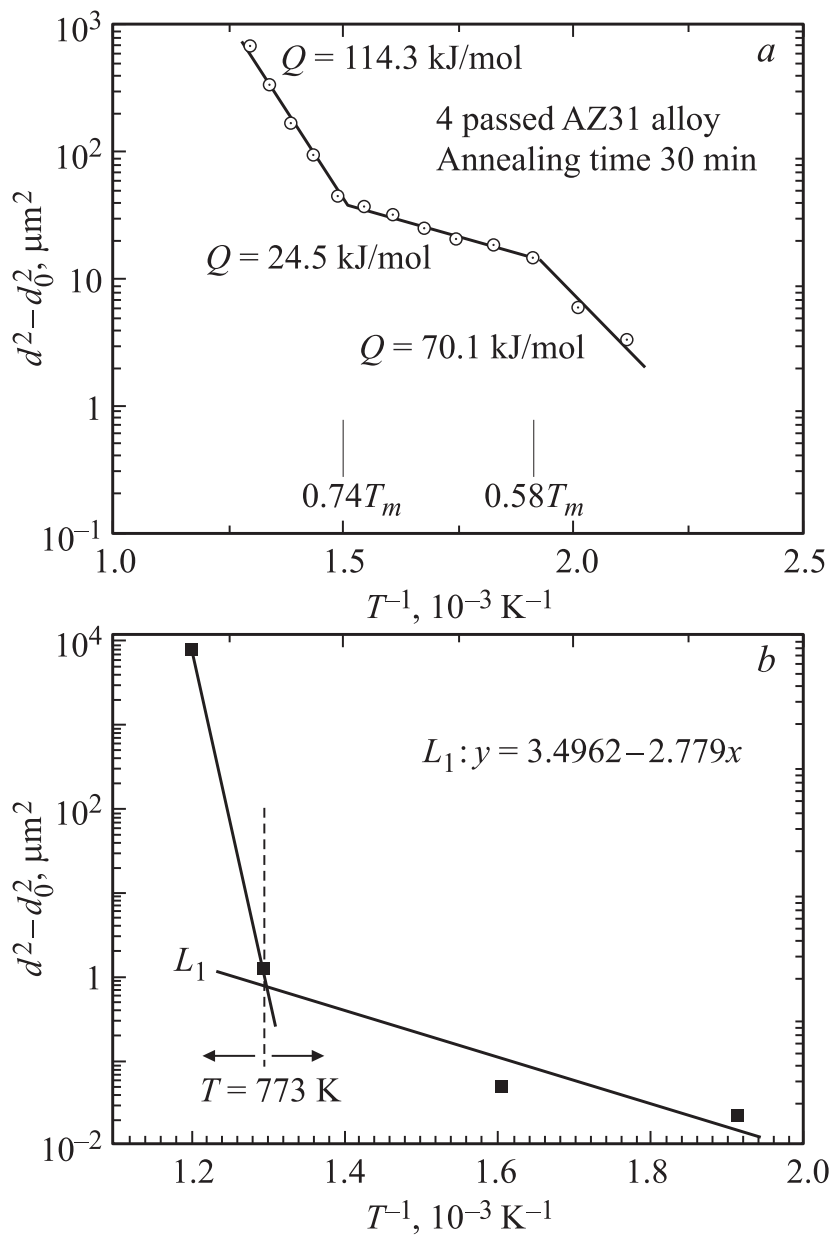

Рис. 2. Зависимость среднего размера зерна от температуры отжига СМК-металлов в полулогарифмических координатах. $a$ - СМК-магниевый сплав Mg-AZ31 [14], $b-$ СМК-алюминиевый сплав $\mathrm{Al}-3.3$ wt.\%Mg-0.2 wt.\%Sc-0.2 wt.\%Zr [17].

В соответствии с подходом, изложенным в $[1,3]$, величина коэффициента зернограничной диффузии в СМК-металлах может быть представлена в виде

$$
D_{b}^{*}=D_{b} \exp \left(\frac{\rho_{b}^{\mathrm{st}} \Delta b+w_{t}^{\mathrm{st}}}{\alpha_{B} w_{0}}\right)
$$

где $\alpha_{B}=0.02$ и $w_{0}=0.1-$ численные коэффициенты $[1,3]$.

Подставляя (5), (6) в (10), получим выражение для $D_{b}^{*}$ в условиях миграции границ (при $V_{m} \geq V_{m}^{*}$ )

$$
D_{b}^{*}=D_{b} \exp \left\{\frac{\left(g_{1} \rho_{v} V_{m}\right)^{1 / 4}+d\left(g_{2} \rho_{v} V_{m}\right)^{1 / 2}}{\alpha_{B} w_{0}}\right\} .
$$

В случае СМК-материалов и при $d<d_{1}$ выражение (11) может быть преобразовано к более простому виду

$$
D_{b}^{*}=D_{b} \exp \left\{\frac{\left(g_{1} \rho_{v} V_{m}\right)^{1 / 4}}{\alpha_{B} w_{0}}\right\} .
$$

Таким образом, скорость миграции границ зерен $V_{m}$ оказывает существенное влияние на диффузионные свойства границ зерен СМК-материалов: при аномальном росте зерен, для которого характерны высокие скорости миграции границ [1,3], а также высоких начальных плотностях решеточных дислокаций, при отжиге СМК-металлов может наблюдаться уменьшение $Q_{b}^{*}$ (этот эффект условно можно назвать эффектом от ускорения зернограничной диффузии), а в условиях собирательной рекристаллизации, при которой наблюдаются заметно меньшие скорости миграции границ [24], величина энергии активации зернограничной диффузии может быть близка к равновесному значению. Отметим, что эффект уменьшения энергии активации роста зерен, который при традиционном анализе данных по рекристаллизации должен проявляться как изменение угла наклона зависимости $\ln \left(d^{2}-d_{0}^{2}\right)-1 / T$, наблюдается довольно часто для широкого круга СМК-материалов $[6,8,14,17]$ (рис. 2).

Перейдем теперь к анализу полученных результатов и их сопоставлению с экспериментальными данными.

2.2. Сопоставление с экспериментом. Для проверки корректности модели рассмотрим результаты экспериментальных исследований рекристаллизации в СМК-меди М0б (99.995\%) и СМК меди М1ф (99.98\%), структура которой сформирована методом равноканального углового прессования (РКУП) (режим $B_{c}$, $T_{\mathrm{ECAP}}=293 \mathrm{~K}$, число циклов прессования $\left.N=8\right)$ [25].

На рис. 3, $a$ представлены зависимости среднего размера зерна $d$ и объемной доли $f_{v}$ материала, охваченного процессом рекристаллизации, от температуры отжига СМК-меди. Как видно из рис. 3, $a$, зависимость среднего размера зерна $d(T)$ имеет трехстадийный характер. На первой стадии отжига (при $T \leq T_{1}$ ) в материале наблюдается незначительный рост зерен. Зеренная структура СМК-металла при этом остается однородной. Начиная с температуры $T \sim T_{1}$ в материале начинается процесс аномального роста зерен [25]. При этом возникает бимодальная структура: появляются крупные зерна, размер которых $d_{\max }$ на порядок превосходит средний размер зерен однородной СМК-матрицы. При увеличении температуры отжига объемная доля $f_{v}$ материала, охваченного процессом аномального роста зерен, увеличивается, и при достижении температуры $T_{2}$ величина $f_{v}$ достигает единицы. При последующем повышении температуры отжига (при $T \geq T_{2}$ ) процесс рекристаллизации принимает обычный характер. Более подробно механизм аномального роста зерен в СМК-материалах описан в [1].

Кинетика роста зерен на первой $\left(T \leq T_{1}\right)$ и третьей $\left(T \geq T_{2}\right)$ стадиях может быть описана с помощью закона собирательной рекристаллизации [24]

$$
d^{2}-d_{0}^{2}=2 \gamma_{b} b \frac{\delta D_{b}^{*}}{k T} t
$$

где $d_{0}$ - начальный размер зерна, $\gamma_{b}$ - энергия границы зерна. 

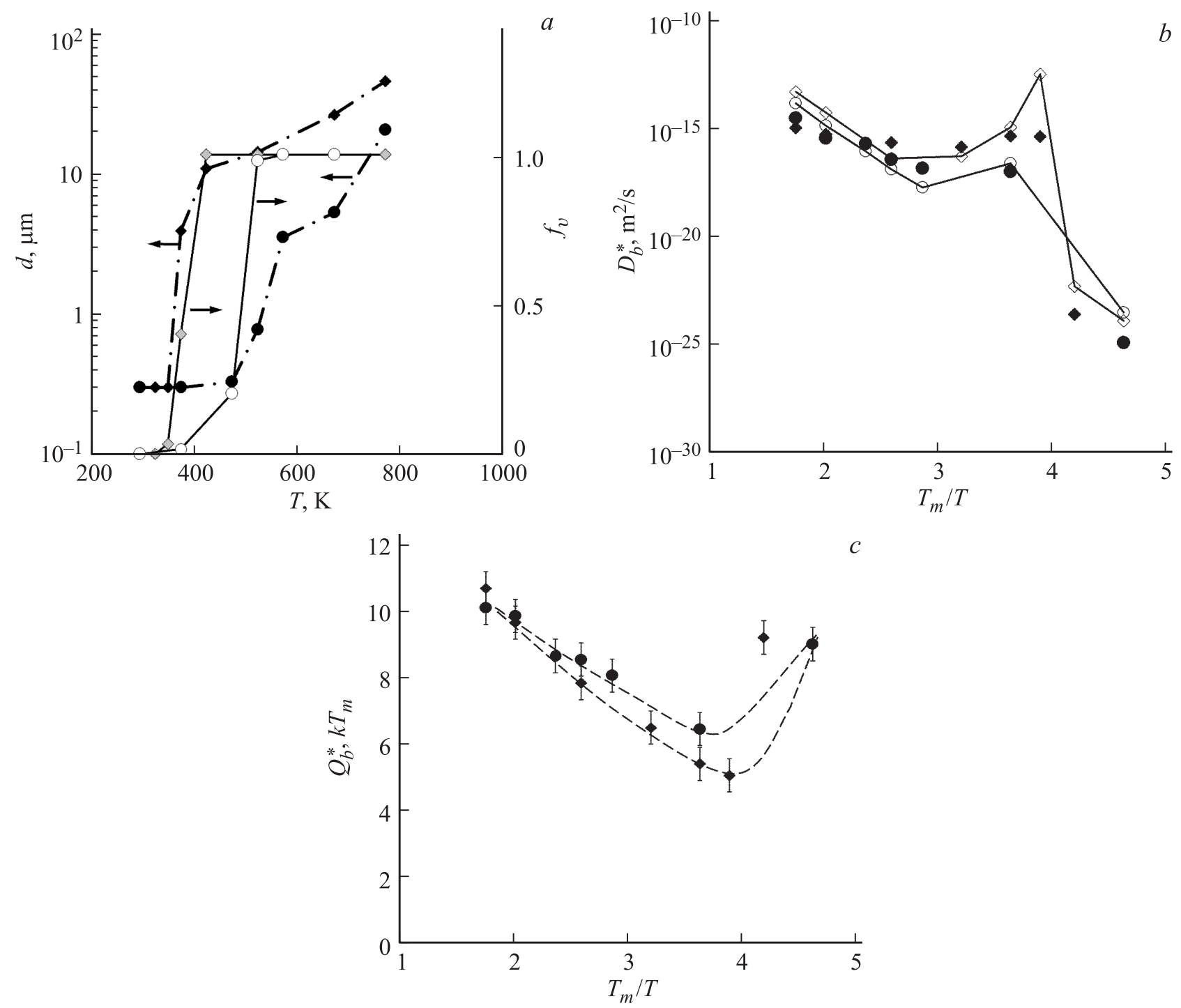

Рис. 3. Зависимость среднего размера зерна $d$ и объемной доли $f_{v}$ материала, охваченного процессом аномального роста зерен [25] (a), а также зависимости коэффициента диффузии $(b)$ и энергии активации зернограничной диффузии $(c)$ от температуры отжига для СМК-меди М0б (99.995\%) и М1ф (99.98\%). Ромбами на всех частях рисунка обозначены данные для СМК-меди М0б (99.95\%), кружками - для СМК-меди М1ф (99.98\%). На части $а$ темные символы - данные по зависимости среднего размера зерна от температуры отжга, светлые - данные по зависимости объемной доли рекристаллизованной структуры от температуры отжига. На части $b$ темные символы - экспериментальные данные, светлые - результаты расчетов.

На стадии аномального роста зерен зависимость среднего размера зерна от времени и температуры отжига может быть рассчитана по формуле $[1,3]$

$$
d=d_{\max }\left(1-\exp \left(-\frac{t}{t_{3}}\right)\right),
$$

где $t_{3}=(d / b)^{2} k T / C_{1} w_{t} D_{b}^{*} \delta G-$ характерное время „ухода“ стыковых дисклинаций, определяющих миграционную подвижность неравновесных границ зерен при отжиге СМК-металлов $[1,3]$.

Воспользовавшись уравнениями (13), (14), можно каждому размеру зерна $d$ на каждой стадии процесса рекристаллизации СМК-материала поставить в соответ- ствие значение $D_{b}^{*}$ (рис. $3, b$ ) и, используя соотношение

$$
Q_{b}^{*}=-\frac{T}{T_{m}} \ln \left(\frac{D_{b}^{*}}{D_{b 0}^{*}}\right)
$$

найти соответствующее значение $Q_{b}^{*}$. Результаты такого расчета представлены на рис. $3, c$.

Из рис. $3, b, c$ видно, что в интервале температур отжига, соответствующих началу аномального роста зерен в СМК-меди М1ф, величина $D_{b}^{*}$ увеличивается от $1.8 \cdot 10^{-24}$ до $\sim 5.5 \cdot 10^{-20} \mathrm{~m}^{2} / \mathrm{s}$; в СМК-меди М0б - от $6.4 \cdot 10^{-24}$ до $4.1 \cdot 10^{-16} \mathrm{~m}^{2} / \mathrm{s}$. В этих же условиях наблюдается уменьшение $Q_{b}^{*}$ : в СМК-меди М1ф величина $Q_{b}^{*}$ уменьшается от исходного значения $\sim(9.0-9.2) k T_{m}$ до значения $\sim 7.6 k T_{m}$, что примерно на 
$\sim 20 \%$ ниже, чем значение энергии активации в равновесных границах зерен $\left(\sim 9.2 k T_{m}\right)$ [22]; в СМК-меди М0б величина энергии активации $Q_{b}^{*}$ уменьшается до $\sim(4.6-5.0) k T_{m}$. При дальнейшем повышении температуры отжига величина $D_{b}^{*}$ немонотонно увеличивается, a $Q_{b}^{*}$ стремится к значениям, характерным для диффузии по равновесным границам зерен. Важно подчеркнуть, что характерные значения $V_{m}$ в СМК-меди лежат в интервале $10^{-8}-10^{-9} \mathrm{~m} / \mathrm{s}$, что более чем на два порядка превышает критическое значение $V_{m}^{*}$ для СМК-меди в рассматриваемом интервале температур (рис. $1, a)$.

Воспользовавшись уравнениями (5), (6), (10) и (12), нетрудно рассчитать зависимость коэффициента зернограничной диффузии СМК-меди от температуры отжига. Результаты расчета зависимости $D_{b}^{*}(T)$ для СМК-меди М1ф и М0б представлены на рис. 3, $b$. Как видно из рисунка, описанная модель дает удовлетворительное согласие теоретических и экспериментальных результатов. Необходимые для расчета значения параметров меди взяты из [3,22,23] (табл. 1).

В работе [6] описаны результаты исследований процесса рекристаллизации алюминия АА1050, СМК-структура в котором (начальный размер зерна $\left.d_{0}=0.35-0.60 \mu \mathrm{m}\right)$ сформирована методом РКУП. Отжиг СМК-образцов проводился в интервале температур 293-773 K (время отжига $t=1 \mathrm{~h}$ ). Авторами [6] отмечается, что зависимость $d(T)$ имеет трехстадийный характер, причем в интервале температур $T=548-573 \mathrm{~K}$ наблюдается аномальный рост зерен, при котором средний размер зерна увеличивается от 0.59 до $3.93 \mu \mathrm{m}$. Дальнейшее повышение температуры отжига $(573-773 \mathrm{~K})$ приводит к плавному повышению размера зерна до $\sim 40 \mu \mathrm{m}$. Проведенный в [6] анализ зависимости $d(T)$ показывает, что на первой и третьей стадии процесса рекристаллизации зависимость $\lg \left(d^{2}-d_{b}^{2}\right)-1 / T$ может быть интерполирована прямыми линиями, причем энергия активации $Q_{b}^{*}$ для стадии низкотемпературного отжига $(T<548 \mathrm{~K})$ составляет $49 \mathrm{~kJ} / \mathrm{mol}\left(\sim 6.2 k T_{m}\right)$, a для стадии высокотемпературной собирательной рекристаллизации при $T>573 \mathrm{~K} Q_{b}^{*}=Q_{b}=85 \mathrm{~kJ} / \mathrm{mol}$ $\left(\sim 10.8 k T_{m}\right)$. Оценка энергии активации для стадии аномального роста зерен авторами [6] не проводилась.

В соответствии с описанной выше процедурой поставим в соответствие каждому размеру зерна на зависимости $d(T, t)$ для СМК-алюминия значения $Q_{b}^{*}$. Проведенная оценка с использованием соотношений (14) и (15) показывает, что на второй стадии отжига (стадия аномального роста) величина $Q_{b}^{*}$ составляет $\sim 5.6 k T_{m}$ $(T=553-573 \mathrm{~K})$.

Заметим, что в материалах, подвергнутых РКУП, не всегда имеет место аномальный рост зерен, а в ряде случаев плотность решеточных дислокаций может быть мала. При этом эффект ускорения зернограничной диффузии может и не наблюдаться, и, как следствие, величина будет $Q_{b}^{*}$ мало меняться в широком диапазоне температур отжига.

\section{3. Эффект повышения коэффициента зернограничной диффуззии при сверхпластической деформации СМК-материалов}

3.1. Описание модели. Как известно, явление сверхпластичности (СП) связано с развитием зернограничного проскальзывания (ЗГП) - особого механизма деформации, который „включается“ при повышенных температурах и особенно эффективно работает в мелкозернистых материалах. Как показано в [26,27], для развития ЗГП необходимы эффективная аккомодация проскальзывания в стыках зерен и неравновесное состояние границ зерен. Выражение для скорости деформации в в условиях СП имеет вид [26]

$$
\dot{\varepsilon}=A\left(\frac{\sigma}{G}\right)^{1 / m}\left(\frac{b}{d}\right)^{p}\left(\frac{G \Omega}{k T}\right) \frac{\delta D_{b}^{*}}{b^{3}}
$$

где $m$ - коэффициент скоростной чувствительности напряжения течения $(\sigma)$.

Для зернограничной деформации $m=0.5$ и $p=2$ [27], но поскольку в условиях СП кроме ЗГП в деформацию вносит вклад и внутризеренное дислокационное скольжение, как правило, в уравнении (16) показатель $m$ меняется от 0.3 до 0.5 [26,27].

Обычно предполагается, что величина $D_{b}^{*}$ при заданной температуре сверхпластичности - постоянная величина, не зависящая от $\dot{\varepsilon}$, и при расчетах скорости деформации по формуле (16) используется равновесное (табличное) значение $D_{b}[26,27]$. При этом, как правило, наблюдается несовпадение теоретических и экспериментальных значений $\dot{\varepsilon}$ : экспериментальная величина скорости деформации оказывается на один-два порядка выше расчетной. Это несоответствие обычно компенсируют вариацией коэффициента $A$ или объясняют нестабильностью зеренной структуры (изменением $d$ в процессе деформации). Однако в ряде случаев различие экспериментальных и расчетных значений столь существенно, что его трудно объяснить неточностью подгоночных параметров. Особенно ярко это несоответствие проявляется в условиях высокоскоростной сверхпластичности СМК-материалов [28].

На наш взгляд, отмеченная проблема может быть решена на основе теории НГЗ [3], в рамках которой можно показать, что в условиях сверхпластической деформации коэффициент зернограничной диффузии может существенно зависеть от скорости деформации [29]. Для того чтобы „уловить“ эту зависимость, необходимо имеющиеся экспериментальные данные по сверхпластичности проанализировать с учетом представлений о зависимости $D_{b}^{*}$ от состояния границ зерен и их свободного объема $[1,3]$. Для этого, подставляя в выражение (16) экспериментально полученные значения $m$, $\dot{\varepsilon}, \sigma, d$ и другие легко измеряемые в эксперименте параметры и принимая $A=$ const, можно определить 
зависимость $D_{b}^{*}(\dot{\varepsilon})$

$$
D_{b}^{*}=\left(\frac{\dot{\varepsilon} b^{2}}{A}\right)\left(\frac{G}{\sigma}\right)^{1 / m}\left(\frac{d}{b}\right)^{p}\left(\frac{k T}{G \Omega}\right) .
$$

Для такого анализа в качестве примера используем результаты исследований высокоскоростной сверхпластичности СМК-сплавов $\mathrm{Al}-3$ wt.\%Mg-0.2 wt.\%Sc [30] и Al-1570 [31].

Необходимые для оценки $D_{b}^{*}$ и $Q_{b}^{*}$ значения переменных $\dot{\varepsilon}, \sigma$ и $m$ приведены в табл. 2 и 3 при $A=100$. Из табл. 2 видно, что в СМК-сплаве $\mathrm{Al}-3$ wt.\% $\mathrm{Mg}-0.2$ wt.\%Sc [30] при увеличении скорости сверхпластической деформации от $10^{-3}$ до $1 \mathrm{~s}^{-1}$ (при $T=673 \mathrm{~K}$ ) имеет место повышение коэффициента зернограничной диффузии примерно на порядок. ${ }^{1}$

В табл. 3 представлены результаты аналогичных оценок величины $D_{b}^{*}$ для СМК-сплава Al-1570 [31]. Из табл. 3 видно, что при $T=723 \mathrm{~K}$ увеличение скорости деформации от $1.4 \cdot 10^{-3}$ до $1.4 \mathrm{~s}^{-1}$ приводит к повышению $D_{b}^{*}$ в $\sim 24$ раза.

Приведенные результаты хорошо согласуются с результатами единственной экспериментальной работы, где проведены непосредственные измерения диффузионной проницаемости границ в условиях структурной сверхпластичности [32]. В этой работе показано, что в сплаве $\mathrm{Zn}-22 \mathrm{wt} . \% \mathrm{Al}$, деформируемом при температуре $\sim 523 \mathrm{~K}$, при изменении скорости деформации от $10^{-4}$ до $10^{-2} \mathrm{~s}^{-1}$ величина коэффициента зернограничной диффузии возросла от $1.28 \cdot 10^{-13}$ до $1.3 \cdot 10^{-11} \mathrm{~m}^{2} / \mathrm{s}[32]$.

Перейдем к объяснению изложенных выше результатов.

Как уже отмечалось, в условиях сверхпластической деформации параметры зернограничной диффузии СМК-металла зависят от параметров потоков решеточных дислокаций, осуществляющих внутризеренную деформацию и бомбардирующих границы зерен, что в свою очередь приводит к появлению на границах зерен стационарной плотности дефектов. Значения $\rho_{b}^{\text {st }} \Delta b$ и $w_{t}^{\text {st }}$ в случае СП имеют вид [1]

$$
\begin{gathered}
\rho_{b}^{\mathrm{st}} \Delta b=\phi_{1} \dot{\varepsilon}_{v}^{1 / 4}, \\
w_{t}^{\mathrm{st}}=\phi_{2} \dot{\varepsilon}_{v}^{1 / 2},
\end{gathered}
$$

где $\phi_{1}$ и $\phi_{2}-$ параметры материала. Подставляя эти выражения в формулу (10), получим

$$
D_{b}^{*}=D_{b} \exp \left(\phi_{1}^{\prime} \dot{\varepsilon}_{v}^{1 / 4}+\phi_{2}^{\prime} \varepsilon_{v}^{\dot{1} / 2}\right),
$$

где $\phi_{1}^{\prime}=\phi_{1} / \alpha_{B} w_{0}, \phi_{2}^{\prime}=\phi_{2} / \alpha_{B} w_{0}$ - численные коэффициенты.

При большой плотности дефектов в границе зерна, возникающей при очень высоких скоростях деформации,

\footnotetext{
${ }^{1}$ При численных оценках не принималась во внимание возможность изменения в условиях ЗГП геометрических характеристик неравновесных границ зерен и, как следствие, их возможное влияние на диффузионные свойства границ зерен при сверхпластической деформации СМК-материалов.
}

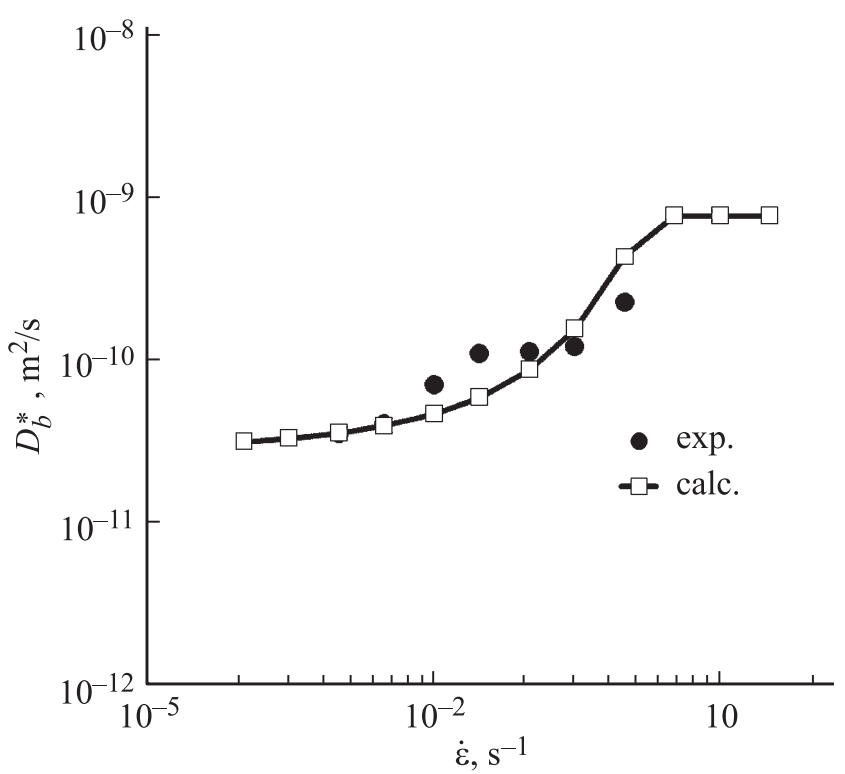

Рис. 4. Численный расчет зависимости коэффициента зернограничной диффузии от скорости сверхпластической деформации СМК-сплава $\mathrm{Al}-3$ wt.\%Mg-0.2 wt.\%Sc. Анализ данных [30].

значение коэффициента диффузии $D_{b}^{*}$ приближается к величине коэффициента диффузии в расплаве $D_{L}$ : $D_{b}^{*}=D_{L}$.

Теоретическая зависимость $D_{b}^{*}\left(\dot{\varepsilon}_{v}\right)$, отражающая поведение, описываемое выражением (20), имеет $S$-образный вид: при малых и больших $\dot{\varepsilon}_{v}$ величина $D_{b}^{*}$ не зависит от скорости деформации, а при промежуточных значени$я \mathrm{x} \dot{\varepsilon}_{v}$ коэффициент зернограничной диффузии интенсивно нарастает с увеличением скорости деформации.

3.2. Сопоставление с экспериментом. Воспользовавшись уравнениями (18)-(20), можно рассчитать зависимость коэффициента зернограничной диффузии от скорости сверхпластической деформации. Результаты расчетов для сплава $\mathrm{Al}-3 \mathrm{wt} . \% \mathrm{Mg}-0.2 \mathrm{wt} . \% \mathrm{Sc}$ представлены в табл. 2 и на рис. 4, для сплава Al-1570 в табл. 3. Расчет для сплава $\mathrm{Zn}-22 \mathrm{wt} . \% \mathrm{Al}(T=523 \mathrm{~K})$ приведен в $[3,28]$.

Необходимые для расчетов значения параметров содержатся в табл. 1.

Как видно из табл. 2 и 3, предложенная элементарная модель удовлетворительно описывает экспериментальные зависимости коэффициента зернограничной диффузии от скорости сверхпластической деформации $D_{b}^{*}\left(\dot{\varepsilon}_{v}\right)^{2}$

\footnotetext{
2 Определенное расхождение результатов численных расчетов с данными работы [30] связано с влиянием деформационно-стимулированного роста зерна: расчет по формуле (20), результаты которого приведены в табл. 2, выполнены при $d_{0}=0.4 \mu \mathrm{m}$. Вместе с тем авторы [30] отмечают, что в процессе испытаний на СП было обнаружено, что в зависимости от температуры и времени деформации средний размер зерна увеличивается до $1-4 \mu \mathrm{m}$. В связи с тем, что в [30] авторы не приводят данных по зависимости $d\left(\dot{\varepsilon}_{v}, T\right)$, а точный расчет этой зависимости настоящее время невозможен, сопоставление результатов расчета с экспериментом проводится при $d_{0}=0.4 \mu \mathrm{m}$.
} 
Таблица 2. Влияние скорости деформации на параметры зернограничной диффузии в СMК-сплаве $\mathrm{Al}-3 \mathrm{wt} . \% \mathrm{Mg}-0.2 \mathrm{wt} . \% \mathrm{Sc}$ $\left(d_{0}=0.4 \mu \mathrm{m}, T=673 \mathrm{~K}\right)$, анализ данных [30]

\begin{tabular}{c|c|c|c|c|c|c}
\hline \multicolumn{3}{|c|}{ Эксперимент $[30]$} & Анализ $[30]$ & \multicolumn{3}{c}{ Расчет } \\
\hline$\dot{\varepsilon}, \mathrm{s}^{-1}$ & $\sigma^{*}, \mathrm{MPa}$ & $m$ & $D_{b}^{*(\exp )} \cdot 10^{11}, \mathrm{~m}^{2} / \mathrm{s}$ & $D_{b 0}^{*} \cdot 10^{6}, \mathrm{~m}^{2} / \mathrm{s}$ & $Q_{b}^{*}, k T_{m}$ & $D_{b}^{*(\text { calc })} \cdot 10^{11}, \mathrm{~m}^{2} / \mathrm{s}$ \\
\hline $1 \cdot 10^{-3}$ & 8 & 0.34 & 52 & 6.76 & 8.8 & 3.45 \\
$3 \cdot 10^{-3}$ & 11 & 0.22 & 4.00 & 6.37 & 8.6 & 3.92 \\
$1 \cdot 10^{-2}$ & 15.5 & 0.37 & 6.96 & 3.49 & 7.6 & 4.63 \\
$3 \cdot 10^{-2}$ & 28 & 0.55 & 10.8 & 2.83 & 7.3 & 8.4 \\
$1 \cdot 10^{-1}$ & 48 & 0.41 & 11.1 & 2.73 & 7.2 & \\
$3 \cdot 10^{-1}$ & 78 & 0.42 & 12.0 & 1.11 & 6.1 & \\
1 & 101 & 0.23 & 22.5 & & & 43.3
\end{tabular}

Таблица 3. Влияние скорости сверхпластической деформации на диффузионные свойства границ зерен в CMК-сплаве Al-1570 $\left(d_{0} \sim 1 \mu \mathrm{m}, T=723 \mathrm{~K}\right)$, анализ данных [31]

\begin{tabular}{c|c|c|c|c|c|c}
\hline \multicolumn{2}{c|}{ Эксперимент [30] } & Анализ $[30]$ & \multicolumn{3}{c}{ Расчет } \\
\hline$\dot{\varepsilon}, \mathrm{s}^{-1}$ & $\sigma^{*}, \mathrm{MPa}$ & $m$ & $D_{b}^{*(\exp )} \cdot 10^{11}, \mathrm{~m}^{2} / \mathrm{s}$ & $D_{b 0}^{*} \cdot 10^{6}, \mathrm{~m}^{2} / \mathrm{s}$ & $Q_{b}^{*}, k T_{m}$ & $D_{b}^{*(\text { calc })} \cdot 10^{11}, \mathrm{~m}^{2} / \mathrm{s}$ \\
\hline $1.4 \cdot 10^{-3}$ & 10 & 0.11 & 2.03 & 7.79 & 9.9 & 2.21 \\
$1.4 \cdot 10^{-2}$ & 13 & 0.23 & 12.1 & 5.27 & 8.3 & 11.8 \\
$5.6 \cdot 10^{-2}$ & 18 & 0.44 & 25.1 & 2.34 & 7.1 & 24.6 \\
$1.4 \cdot 10^{-1}$ & 27 & 0.34 & 27.9 & 2.01 & 6.9 & 27.4 \\
$5.6 \cdot 10^{-1}$ & 43 & 0.45 & 44.1 & 0.9 & 6.0 & 41.7 \\
1.4 & 65 & 0.45 & 48.2 & 0.8 & 5.8 & $\mathbf{4 5 . 8}$
\end{tabular}

\section{4. Выводы}

1. Разработана модель изменения диффузионных свойств неравновесных границ зерен при рекристаллизации СМК-металлов. Показано, что необходимым условием для повышения коэффициента зернограничной диффузии при отжиге СМК-металлов является аномальный рост зерен, характеризующийся повышенной скоростью миграции границ зерен.

2. Разработана модель ускорения зернограничной диффузии при сверхпластической деформации СМК-материалов. Установлено, что условием, необходимым для повышения коэффициента зернограничной диффузии при сверхпластичности СМК-материалов, являются высокая скорость внутризеренной деформации и высокая плотность решеточных дислокаций.

Результаты численных расчетов, проведенных в рамках разработанных моделей, хорошо согласуются с литературными данными.

\section{Список литературы}

[1] V.M. Segal, I.J. Beyerlein, C.N. Tome, V.N. Chuvil'deev, V.I. Kopylov. Fundamentals and engineering of severe plastic deformation. Nova Science Publ., N. Y. (2010). 549 p.

[2] В.Н. Чувильдеев, О.Э. Пирожникова, А.В. Петряев. ФММ 92, 6, 14 (2001).

[3] В.Н. Чувильдеев. Неравновесные границы зерен в металлах. Теория и приложения. Физматлит, М. (2004). 304 с.
[4] S.V. Divinski, G. Reglitz, H. Rösner, Y. Estrin, G. Wilde. Acta Mater. 59, 1974 (2011).

[5] S.V. Divinski, G. Reglitz, I.S. Golovin, M. Peterlechner, R. Lapovok, Y. Estrin, G. Wilde. Acta Mater. 82, 11 (2015).

[6] C.Y. Yu, P.L. Sun, P.W. Kao, C.P. Chang. Mater. Sci. Eng. A 366, 310 (2004).

[7] H.-K. Kim. J. Mater. Sci. 39, 7107 (2004).

[8] J. Stráská, M. Janeček, J. Čižek, J. Stráský, B. Hadzima. Mater. Charact. 94, 69 (2014).

[9] X. Molodova, G. Gottstein, M. Winning, R.J. Hellmig. Mater. Sci. Eng. A 460-461, 204 (2007).

[10] V.N. Chuvil'deev, T.G. Nieh, M.Yu. Gryaznov, A.N. Sysoev, V.I. Kopylov. J. Alloys. Compd. 378, 253 (2004).

[11] R. Kapoor, J.K. Chakravartty. Acta Mater. 55, 5408 (2007).

[12] T. Fujita, Z. Horita, T.G. Langdon. Mater. Science Eng. A 371, 241 (2004).

[13] S.V. Divinski, J. Ribbe, D. Baither, G. Schmitz, G. Reglitz, H. Rösner, K. Sato, Y. Estrin, G. Widle. Acta Mater. 57, 5706 (2009).

[14] H.K. Kim, W.J. Kim. Mater. Sci. Eng. A 385, 300 (2004).

[15] Ю.Р. Колобов, Г.П. Грабовецкая, К.В. Иванов, Н.В. Гирсова. ФММ 91, 5, 107 (2001).

[16] В.Н. Чувильдеев, В.И. Копылов, М.Ю. Грязнов, А.Н. Сысоев, Б.В. Овсянников, А.А. Флягин. ДАН 423, 334 (2008).

[17] H.B. Geng, S.B. Kang, B.K. Min. Mater. Sci. Eng. A 373, 229 (2004).

[18] М.Ю. Грязнов, А.Н. Сысоев, В.Н. Чувильдеев. ФММ 87, 2, 84 (1999).

[19] И.С. Головин. ФММ 110, 424 (2010).

[20] D. Prokoshkina, L. Klinger, A. Moros, G. Wilde, E. Rabkin, S.V. Divinski. Acta Mater. 69, 314 (2014). 
[21] В.Н. Чувильдеев. ФММ 81, 5, 5 (1996).

[22] Г.Дж. Фрост, М.Ф. Эшби. Карты механизмов деформации. Металлургия, Челябинск. (1989). 328 с.

[23] А.Р. Уббелоде. Расплавленное состояние вещества. Металлургия, М. (1982). 376 с.

[24] С.С. Горелик, С.В. Добаткин, Л.М. Капуткина. Рекристаллизация металлов и сплавов. МИСИС, М. (2005). 432 с.

[25] А.В. Пискунов, В.Н. Чувильдеев, Ю.Г. Лопатин. В сб.: ВНКСФ-15. АСФ России, Екатеринбург-Кемерово (2009). C. 759 .

[26] T.G. Nieh, D. Wadsworth, O.D. Sherby. Superplasticity in metals and ceramics. Cambridge Univ. Press, Cambridge (1997). 316 p.

[27] V.N. Perevezentsev, V.V. Rybin, V.N. Chuvil'deev. Acta Met. Mater. 40, 887 (1992).

[28] T.G. Langdon. J. Mater. Sci. 44, 5998 (2009).

[29] В.Н. Чувильдеев, А.В. Петряев. ФММ 89, 2, 24 (2000).

[30] M. Furukawa, A. Utsunomiya, K. Matsubara, Z. Horita, T.G. Langdon. Acta Mater. 49, 3829 (2001).

[31] F. Musin, R. Kaibyshev, Y. Motohashi, G. Itoh. Scripta Mater. 50, 511 (2004).

[32] С.В. Земский, Н.Е. Фомин, Г.К. Мальцева. ФХОМ 4, 91 (1978). 